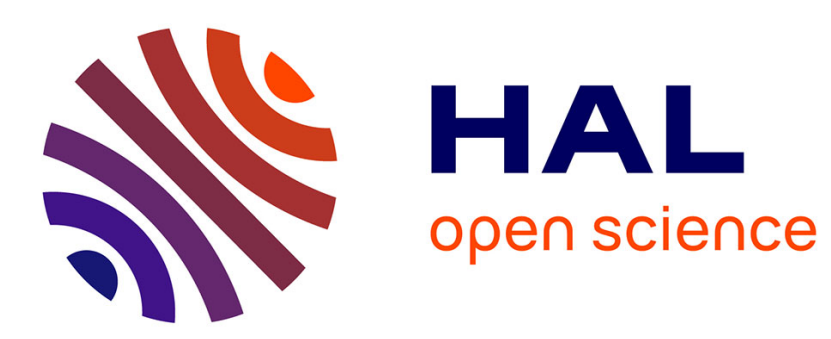

\title{
THE TRANSPORT COEFFICIENT FOR NON-TRANSITION ELEMENTS
}

\author{
Dj. Jovik, M. Davidovik, D. Chaturvedi
}

\section{To cite this version:}

Dj. Jovik, M. Davidovik, D. Chaturvedi. THE TRANSPORT COEFFICIENT FOR NONTRANSITION ELEMENTS. Journal de Physique Colloques, 1980, 41 (C8), pp.C8-276-C8-279. 10.1051/jphyscol:1980870 . jpa-00220523

\section{HAL Id: jpa-00220523 https://hal.science/jpa-00220523}

Submitted on 1 Jan 1980

HAL is a multi-disciplinary open access archive for the deposit and dissemination of scientific research documents, whether they are published or not. The documents may come from teaching and research institutions in France or abroad, or from public or private research centers.
L'archive ouverte pluridisciplinaire HAL, est destinée au dépôt et à la diffusion de documents scientifiques de niveau recherche, publiés ou non, émanant des établissements d'enseignement et de recherche français ou étrangers, des laboratoires publics ou privés. 


\title{
THE TRANSPORT COEFFICIENT FOR NON-TRANSITION ELEMENTS
}

\author{
Dj. Jovič, M. Davidovič and D.K. Chaturvedi* \\ Boris Kidrǐ̌ Institute of Nuclear Sciences, Vinča, Belgrade, Yugoslavia
}

\begin{abstract}
Using the recent experimental data for 1iquid aluminum, copper, lead, tin, gallium and bismuth, the effective interatomic potential $\varphi(r)$ has been calculated by inverting the Week-Chandler-Anderson (WCA) perturbation theory.Also, the first and second derivatives of interatomic potential $\varphi(r)$, along with different correlation functions as $g(r), c(r)$, have been calculated for the mentioned liquid metals. With knowledge of the above quantities and their products, different transport coefficients, such as self-diffusion $D$, viscosity $n$ and conductivity $t$ have been studied, for the group of transition and non- transition elements, and their results compared with classical measurements of the macroscopic quantities.
\end{abstract}

Our aim in this work was to study the transport processes in the transition and non-transition elements from the aspects of their microscopic static properties. The transport coefficients $\mathrm{D}$ can be defined in terms of the intermolecular forces through the relations of Rice and Kirkwood /1-3/,

$$
\begin{aligned}
& D=\frac{K_{B} T}{J} \\
& J^{2}=\frac{4 \pi}{3} n \cdot m \int_{0}^{-} r[r \ddot{\varphi}(r)+2 \dot{\varphi}(r)] g(r) d r
\end{aligned}
$$

Here $\ddot{\varphi}(r)$ and $\dot{\varphi}(r)$ denote the derivatives of the effective pair potential $\varphi(r), g(r), m, n$ are respectively the pair correlation function, mass and density of the system. In a similar manner the shear viscosity coefficient $\eta$, the bulk viscosity $\eta_{v}$ and the coefficient of the conductivity can be expressed

$$
\begin{aligned}
& \text { as /3-4/ } \\
& \eta=\frac{2 \pi}{15} \frac{n^{2} m}{\xi} \int_{0}^{\infty} r^{4}\left[\ddot{\varphi}(r)+\frac{4}{r} \dot{\varphi}(r)\right] g(r) d r \\
& \eta_{v}=\frac{2 \pi}{9} \frac{n^{2} m}{\xi} \int_{0}^{\infty} r^{4}\left[\ddot{\varphi}(r)+\frac{1}{r} \dot{\varphi}(r)\right] g(r) d r \\
& \text { and } \\
& A=-\frac{\pi}{3} \frac{K_{B} T}{J} \frac{\partial}{\partial T}\left[(1-N) \frac{n}{V} \int_{0}^{\infty} r^{4} \ddot{P}(r) g(r) d r\right]
\end{aligned}
$$

From the above equations, it can be shown that all the transport properties can be obtained if the shape of the effective interatomic potential $\varphi(r)$ is known.

In the present work we extract $\varphi(r)$ from the measured data of the structure factor $S(q)$. The pro- blem is of fundamental importance and many different theoretical approaches were used in order to study this problem. In this paper we use an inverted perturbation scheme of Week"s, Chandler and Anderson /5-6/ (recently used by Mitra /7/ for liquid Ne and $\mathrm{Pb})$ to derive the effective pair potential $\varphi(r)$ for six liquid metals, namely copper $/ 8 /$, aluminum $/ 9 /$, gallium $/ 10 /$, tin $/ 11 /$, lead $/ 12 /$ and bismuth $/ 13 /$ since we had a recent structure data of those metals for several temperatures. However, due to limitations of space we shall present here only the results for copper and aluminum.

In WCA approximation, $\varphi(r)$ is divided into a short-range repulsive $\varphi_{S}(r)$ and long-range attractive part $\varphi_{l}(r)$ as

$$
\varphi(r)= \begin{cases}\varphi_{S}(r), & r \leqslant r_{0} \\ \varphi_{l}(r), & r>r_{0}\end{cases}
$$

where $r_{0}$ is the distance at which $\varphi(r)$ has a minimum. Then instead of calculating the actual system directly, one starts with a trial ( $T$ ) system with hard spheres fluid as the reference system and treats the effect of attractive interaction as a small perturbation to this reference system. Thus the trial pair potential can be written as

$$
\varphi_{T}(r)=\varphi_{d}(r)+\varphi_{\ell}(r)
$$

where $\varphi_{d}(r)$ is the hard sphere potential, $d$ being the hard core diameter. The trial pair potential is made *) I.C.T.P.,P.O.B. 586 Miramare,
341000 Trieste, Italy 
close to the actual pair potential by choosing the hard core diameter through an optimized method. Therefore this method is also applicable to systems - like liquid metals, where long range part of the potential is a large one.

Our approach differs from that of Mitra /14/ who used random phase approximation (RPA) for the structure factor of the trial system. As it is well known, RPA does not include the effect of correlation between atoms which is important in determination of the structure factor at small wave vectors. The shape of $S(q)$ and the location of the first peak $\left(q_{0}\right)$ have very different effects on the pair potential of a liquid state. For example, shape of $S(q)$ for $q>q_{0}$ as well as the position of first peak (but not its height) determinate the repulsive part of the potential, especially for high densities. On the contrary, values of $S(q)$ for $q<q_{0}$ and the height of the first peak (but not its affect to the long-range part of the pair potential $\varphi_{\ell}(r)$. Therefore, we consider a trial structure $S_{J}(q)$

$$
S_{T}(q)=\frac{S d(q)}{\left[1+n \beta \psi_{l}(q) S_{d}(q)\right]}
$$

where $S_{d}(q)$ is the hard sphere structure factor and $\Psi_{l}(q)$ is the Fourier transform of the effective atractive field $\psi_{\ell}(r)$. Recently, Evans and Schirmacher /15/ and also Gaskell /16/ have tried to calculate $\psi_{l}(q)$ through a perturbation procedure. In this work we use the expression due to Gaskell /16/

$$
\beta^{-1} \Psi_{l}(q)=\varphi_{l}(q)+\frac{1}{(2 \pi)^{3} n} \int_{0}^{\infty} d q^{9^{3}} \varphi_{l}\left(q^{3}\right) S_{d}^{2}\left(q^{2}\right)\left[S_{d}\left(1 q^{g}+q\right)-1\right](q)
$$

where $s_{d}(q)$ was replaced by $S_{\exp }(q)$.

In order to calculate $S_{T}(q)$ from Eqs. (8) and

(9) the hard sphere structure factor and the attractive part of the pair potential is needed. For $S_{d}(q)$, we use A shcroft and Lekner /17-18/ solution of the PercusYevick equation of the hard sphere structure factor and for $\varphi_{\ell}(r)$ we make the ansatz

$$
\beta \varphi_{l}(r)=-c(r), \quad r>\alpha
$$

where $c(r)$ is the direct correlation function. In the physically inaccessible region, $r<d, \varphi_{l}(r)$ is assumed of the form /19/

$$
\beta Y_{l}(r)=A+(1-u) B+(1-u)^{2} \sum_{n=0}^{\infty} C_{n} P_{n}(2 u-1)
$$

where $P_{n}$ are the Legendre Polynomials and $u=\frac{r}{d}$. The physical reasons for choosing such a form and the determination of the parameters $A, B, C_{n}$, etc. have already been discussed by WCA and Ailawadi et al. /19-20/. The pair correlation function of the real system - liquid metal is now obtained by the approximation

$$
g(r)=\exp [-\beta \varphi(r)] Y_{T}(r)
$$

where $Y_{T}(r)$ is related to the pair correlation function of the trial system

$$
Y_{T}(r)=\exp \left[\beta \varphi_{T}(r)\right] g_{T}(r)
$$

Finally, we obtained an optimum value of the hard core diameter $d$, using the condition

$$
\int_{0}^{\infty} g(r) d T^{3}=\int_{0}^{\infty} g_{T}(r) d T^{3}
$$

\section{Numerical procedure}

Due to experimental limits the structure factor can not be precisely measured both for small and large wave vectors. Therefore, we extrapolate $S_{\exp }(q)$ to the exact value of $S(0)$, determined by the isothermal compressibility $K_{T}$ as

$$
S(0)=\frac{n}{\beta} K_{T}
$$

For $q>q_{\max }$ (max.measured $q$ ), we used the expression

$$
c(q)=c_{1}+c_{2} \cos \left[c_{3} q-c_{4}\right] \exp \left[-c_{5} q\right]
$$

Parameters $C_{n} ; n=1, \ldots, 5$ are determined by the least square fit of eq. (16) with $s_{\exp .}(q)$ for q greater than the first minimum position. We also defined $S_{\text {exp }}(q)$ for $o \leqslant q \leqslant 20 \AA^{-1}$, which is sufficient to obtain $\mathrm{q}(x)$ by the Fourier transform.

For numerical calculations of a transport coefficients we started with the Aschcroft-Lekner expression for the hard sphere structure factor. The initial values of the hard core diameters, now denoted as $\mathrm{d}^{(1)}$, are obtained for all liquid metals by comparing the $S_{d}(q)$ with the respective $S_{\exp }(q)$ through the last square method. The next step is to obtain $\varphi_{\ell}(q)$ from Eqs. (10) and (11). This requires the data of $c(r)$, for which we use the relation

$$
c(r)=\left[2 \pi^{2} n r\right]^{-1} \int_{0}^{2}\left[1-\frac{1}{s(2)}\right] 2 \sin 2 r d q
$$


Then we tested its accuracy through another compressibility equation

$$
\frac{\beta}{n K_{T}}=1-4 \pi n \int_{0}^{\infty} c(r) r^{2} d r
$$

By means of Eqs. (8) and (9) we obtained $S_{T}(q)$, and its Fourier transform $g_{T}(r)$. By substitution of $g_{T}(r)$ and $\varphi_{T}(r)$ in (13) and $\varphi_{T}(r)$ in (12) one gets a pair potential, denoted as $\varphi^{(2)}(r)$, corresponding to $g_{\text {exp }}(r)$.. Finally, the condition (14) determinates an improved value of the hard-core diameter $d^{(2)}$, where $\varphi^{(2)}(r)$ is again split up as described in eq. (6) to obtain a new $\varphi_{l}^{(2)}(r)$. With new values of $\mathrm{S}_{\mathrm{d}}{ }^{(2)}(\mathrm{q})$ (corresponding to $\mathrm{d}^{(2)}$ and $\varphi_{\ell}{ }^{(2)}(q)$ ) as input, we calculate $S_{T}{ }^{(2)}(q)$ and repeated the whole procedure until a satisfactory convergence for $\varphi(r)$ is achieved.

After this, the first and the second derivatives of the potential with the pair correlation function determinate the transport coefficients as given in Eqs. (1) - (5).

\section{Results and Discussion}

By the above procedure we calculated the effective interaction potential in liquid copper and aluminum. The experimental structure factor data $S_{(q)}$ for liquid copper have been measured by O.Eder, $B$. Kunsch et al. (1979), at two temperatures: $1393 \mathrm{~K}$ and $1833 \mathrm{~K}$. The structure factor $\mathrm{S}(\mathrm{q})$ for liquid aluminum has also been measured by $\mathrm{Dj}$. Jović et al. (1977) at two temperatures: $939 \mathrm{~K}$ and $1073 \mathrm{~K}$. The structure factor data both for $\mathrm{Cu}$ and $\mathrm{Al}$ were extended up to $20 \AA^{-1}$ in order to get more accurate Fourier transform to calculate the correlation functions. For long wave vectors of momentum transfer the variation of $S(q)$ is small and less than $0.1 \%$. A ccording to this procedure the ripples in $g(r)$ for small $r$ almost disappear. The calculated direct correlation function $c(r)$ shows very small fluctuations for large $r$ but $c(r)$ exhibits small positive values when $r$ increases. This means that a long-range attractive part of $\varphi_{l}(r)$ is also small; pronounced bumps disappear through iteration process. To get the short-range component of $\varphi_{l}(r)$ one has to solve a linear integral equation of the Fredholm type. This equation has to be converted into a system of linear equations /21/

$$
\left[\Omega_{i}^{0}\right]=\left[\delta_{i j}+G_{i j} h_{j}\right]\left[\lambda_{j}\right]
$$

where $\lambda_{i}$ and $\lambda_{j}$ are variables proportional to the pair potential $\varphi_{\ell}(r)$ in RPA and GRPA approximation respectively. The matrix element $G_{i j}$, i.e. the kermal of Fredholm's equation $G\left(q^{\prime}, q\right)$ is expressed as

$$
G\left(q^{\prime}, q\right)=\frac{1}{4 \pi^{2} n} S \exp \left(q^{\prime}\right) \int_{\mid q^{\prime}-2 !}^{2^{\prime}+q} x[S \exp (x)-1] d x
$$
where $h_{j}$ is a variable of the integration interval. A special program was used for solving the matrix equation (19). The details of this program and the results will be presented in the next paper.

In the Figs. 1 and 2 is shown, for two different temperatures the pair interaction potential $\varphi(r)$. Both figures show the expected behaviour for data at higher temperature where potential $\varphi(r)$ is softer and the steepness is smaller than near the melting point.

As it can be seen from Eqs. 1-5 the transport coefficients generally depend on the shape of $\dot{\varphi}(r)$ and $\ddot{\varphi}(r)$ which again are sensitive on the profile. of $\varphi(r)$.

Table 1 contains numerically calculated transport coefficients and compared with experimental ones for two metals and given temperatures.

Since we have data for two temperatures only, the value of the thermal conductivity coefficient sho-

\begin{tabular}{|c|c|c|c|c|c|c|c|c|c|}
\hline \multirow{2}{*}{ METAL } & \multirow{2}{*}{$\begin{array}{c}T \\
|K|\end{array}$} & \multicolumn{2}{|c|}{$\mathrm{D}\left[\mathrm{cm}^{2} / \mathrm{sEc}\right]$} & \multicolumn{2}{|c|}{$n_{[}[\mathrm{CP}]$} & \multicolumn{2}{|c|}{$n_{v}\{c P\}$} & \multicolumn{2}{|c|}{$=$ LWATT $\left.\mathrm{cm}^{-1} \mathrm{~K}^{-1}\right)$} \\
\hline & & $C_{A L}$. & Exp. & CAL. & Exp. & CAL. & Exp. & CAL. & Exp. \\
\hline \multirow{2}{*}{ Cu } & 1393 & 6.53 & 4.22 & 1.13 & 3.8 & 2.405 & 6.3 & 1.39 & 1.1 \\
\hline & 1833 & 7.7 & 9. & 1.05 & 2.6 & 2.30 & 4,3 & 1.56 & 1.15 \\
\hline \multirow{2}{*}{ AL } & 939 & 1.8 & 1.71 & 1.35 & 1.2 & 2.01 & 2.0 & 2.08 & 1.2 \\
\hline & 1073 & 5.4 & 7.1 & 0.7 & 0.95 & 1.403 & 2.6 & 0.7 & 0.9 \\
\hline
\end{tabular}
uld be considered as a preliminary one.

Table 1 


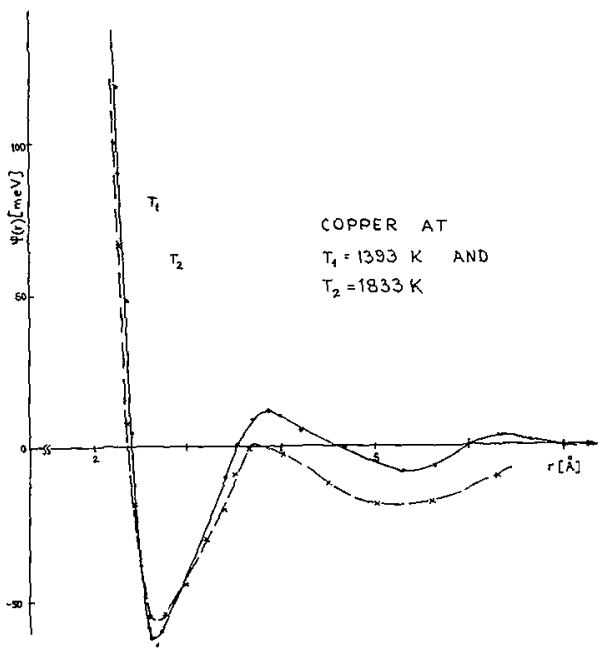

Fig. 1

It should be pointed out that transport coefficients are very sensitive to the values of $S(q)$ at low $q$, what has been improved in calculation by GRPA. The differences in Table 1 between calculated and experimental values are caused by neglection of electron contribution.

\section{References}

1 Rice, A.S., Kirkwood, G.J., J.Chem.Phys., 31 (1959) 901.

2 Bansal, R., J.Phys. C. 6 (1973) 1201 and 3071

3 Eder, J.O., Kunsch, B., Presse, G., Sonderdruck aus 52. Heft 6 (1976) 319.

4 Croxton, C., Introduction to Liquid State Physics, John Wiley and Sons, London, 1975.

5 Weeks, D.J., Chandler, D. and Andersen, C.H., J.Chem.Phys., 55 (1971) 5422.

6 Andersen, C.H., Chandler,D.J. and Weeks, D. J. J. Chem. Phys., 57 (1972) 2626.

7 Mitra, K.S., J.Phys. C. 10 (1977) 2033.

8 Kunsch, B. (1979) (private communication).

9 Jović, Dj., Padureanu, I. and Rapeanu, S., Inst. Phys.Conf.No. 30, Chap. 1, Part 1, 1 (1977) 120. 10 Bellissent, R. (1979) (private communication).

11 Jović, Dj, and Padureanu, I., (1978)

(unpublished data)

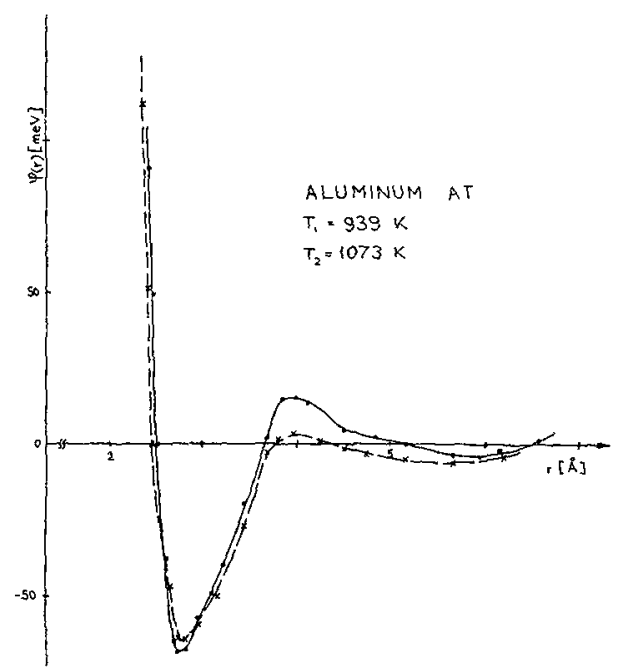

Fig. 2

Acknowledgement

The authors wish to thank to Drs. T. Gaskell, U. Dahlborg and I. Ebbsjo for the useful discussions and suggestions. Also, Dr. M.Davidović expresses his gratitude to ICTP at Trieste for the hospitality during his work at the Computer Centre.

12 Dahlborg, U., Davidović, M. and Larsson, K.E., Phys.Chem. Liquid 6 (1977) 149.

13 Lampater, P. (1979) (private communication). 14 Mitra, K.S. and Gillan, J. M., Phys. C. 9 (1976) 1515.

15 Evans, R. and Schirmacher, W., J.Phys. C 11 (1978) 2437.

16 Gaskell, T., Phys. Lett. 65A (1978) 421.

17 A shcroft, W.N. and Langreth, C.D., Phys. Rev. 159 (1967) 500.

18 Davidović, M., PhD Thesis, Belgrade University, (1978) (unpublished).

19 Ailawadi, K.N., Miller, E.D. and Naghizadeh, J., Phys.Rev. Lett.36 (1976) 1494.

20 Ailawadi, K. N., Phys. Reports 57.4 (1980) 241.

21 Delves, M.L., and Walsh, J., eds., Numerical Numerical Solution of Integral Equations, Clarendon Press, Oxford, 1974. 